Edith Cowan University

Research Online

ECU Publications 2011

$1-1-2011$

\title{
Spatially resolved inhomogeneous depressions of the excitons Zeeman splitting in an integrated magnetic-multiple quantum wells system
}

\author{
Ahmed Abdelrahman \\ Edith Cowan University \\ Hoon Soo Kang \\ Sang Youp Yim \\ Mikhail Vasiliev \\ Edith Cowan University \\ Kamal Alameh \\ Edith Cowan University
}

See next page for additional authors

Follow this and additional works at: https://ro.ecu.edu.au/ecuworks2011

Part of the Physical Sciences and Mathematics Commons

$10.1063 / 1.3603000$

This is an Author's Accepted Manuscript of: Abdelrahman, A. , Kang, H., Yim, S., Vasiliev, M. , Alameh, K. , \& Lee, Y. T. (2011). Spatially resolved inhomogeneous depressions of the excitons Zeeman splitting in an integrated magneticmultiple quantum wells system. Journal of Applied Physics, 110(1), 5. Available here This Journal Article is posted at Research Online. https://ro.ecu.edu.au/ecuworks2011/101 


\section{Authors}

Ahmed Abdelrahman, Hoon Soo Kang, Sang Youp Yim, Mikhail Vasiliev, Kamal Alameh, and Yong Lee 


\title{
AIP Aappied Physics
}

\section{Spatially resolved inhomogeneous depressions of the excitons Zeeman splitting in an integrated magnetic-multiple quantum wells system}

\author{
A. Abdelrahman, H. Kang, S. Y. Yim, M. Vasiliev, K. Alameh et al.
}

Citation: J. Appl. Phys. 110, 013710 (2011); doi: 10.1063/1.3603000

View online: http://dx.doi.org/10.1063/1.3603000

View Table of Contents: http://jap.aip.org/resource/1/JAPIAU/v110/i1

Published by the American Institute of Physics.

\section{Related Articles}

Polarization spectroscopy of N-polar AIGaN/GaN multi quantum wells grown on vicinal (000) GaN Appl. Phys. Lett. 101, 182103 (2012)

Negative residual infrared photoconduction in the $\mathrm{p}$-SiGe/Si heterostructures with selectively doped quantum wells

J. Appl. Phys. 112, 083715 (2012)

Transient effects of pump-probe optical response in intersubband transitions of semiconductor quantum wells J. Appl. Phys. 112, 073117 (2012)

Optical absorption in semiconductor quantum dots coupling to dispersive phonons of infinite modes

J. Appl. Phys. 112, 074324 (2012)

Two-dimensional distributed-feedback in InGaAs/GaAs quantum structure lattice arrays

Appl. Phys. Lett. 101, 141127 (2012)

\section{Additional information on J. Appl. Phys.}

Journal Homepage: http://jap.aip.org/

Journal Information: http://jap.aip.org/about/about_the_journal

Top downloads: http://jap.aip.org/features/most_downloaded

Information for Authors: http://jap.aip.org/authors

\section{ADVERTISEMENT}
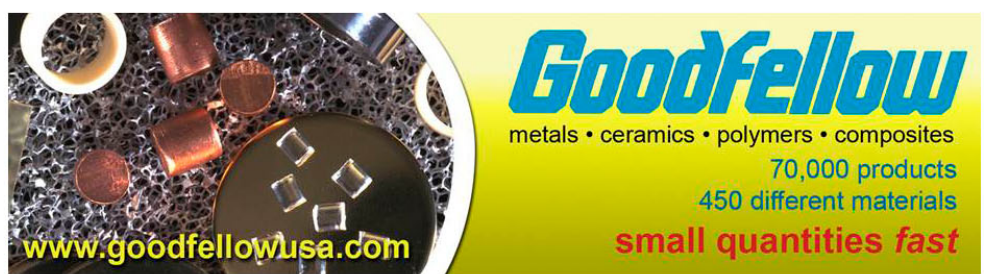


\title{
Spatially resolved inhomogeneous depressions of the excitons Zeeman splitting in an integrated magnetic-multiple quantum wells system
}

\author{
A. Abdelrahman, ${ }^{1, a)}$ H. Kang, ${ }^{2, a)}$ S. Y. Yim, ${ }^{2}$ M. Vasiliev, ${ }^{1}$ K. Alameh, ${ }^{1,3}$ and Y. T. Lee ${ }^{3}$ \\ ${ }^{1}$ Electron Science Research Institute, Edith Cowan University, 270 Joondalup Drive, \\ Joondalup WA 6027 Australia \\ ${ }^{2}$ Quantum Optics Laboratory, Advanced Photonics Research Institute, Gwangju Institute of Science and \\ Technology, 261 Cheomdan-gwagiro (Oryong-dong), Buk-gu, Gwangju 500-712, South Korea \\ ${ }^{3}$ Department of Nanobio Materials and Electronics, Gwangju Institute of Science and Technology, \\ 261 Cheomdan-gwagiro (Oryong-dong), Buk-gu, Gwangju 500-712, South Korea
}

(Received 20 December 2010; accepted 17 May 2011; published online 8 July 2011)

\begin{abstract}
We report on the generation of a nonuniform spatial distribution of the heavy and light-hole excitons in a multiple quantum wells system integrated with a localized inhomogeneous weak magnetic field. An inhomogeneous spatially resolved depression of the Zeeman splittings of the heavy-hole excitons and the light-hole excitons with respect to their translational wave vectors is observed. A localized inverted concentration of the two types of the excitons due to the inhomogeneity of the magnetic field is also measured. A simple method to integrate permanent magnetic materials with the multiple quantum wells system is used to create an accessible degree of control for magnetically manipulating the excitonic distributions. (C) 2011 American Institute of Physics. [doi:10.1063/1.3603000]
\end{abstract}

\section{INTRODUCTION}

Studying the behavior of the bound state of the composite electron-hole particles, known as excitons, when under the influence of an external magnetic field reveals very interesting underlying physics and enriches our understanding of the basic concepts of such quantum systems. ${ }^{1}$ Among these interesting results is the observation of a remarkable enhancement in the magnetic moments of excitons caused by their motion in an external uniform strong magnetic field. ${ }^{2}$ It has also been observed that the Zeeman splitting and the diamagnetism properties of the excitons are functions of the exciton translational wave vector, which itself is a function of an external magnetic field applied in a specific direction. ${ }^{3}$ Most importantly, both the excitons energy splitting and their effective masses are found to depend strongly on the magnitude of the external magnetic field and its direction. At high fields, the effective masses of the excitons become larger than the sum of the electron and hole masses. ${ }^{4}$

Combining magnetic materials with quantum well systems has been proposed theoretically and demonstrated experimentally to achieve a localized magnetic field control of the translational wave vector of the excitons. ${ }^{5-7}$ For example, J. Levy et al. ${ }^{8}$ were able to measure the effect of systematically distributed magnetic ions $\left(\mathrm{Mn}^{2+}\right)$ on the spin behavior of the excitons created in $\mathrm{ZnSe} / \mathrm{Zn}_{0.80} \mathrm{Cd}_{0.20} \mathrm{Se}$ quantum wells, which had specific magnetic-ion patterns implanted using a focused beam of $\mathrm{Ga}^{+}$ions. Moreover, the effect of the magnetic field is thought to enhance the excitons concentration, as shown previously by Lerner, Lozovik, Kuramoto, and Horie, where strong magnetic field perpendicular to the well plane is important to improve the critical condi-

\footnotetext{
a) Author to whom correspondence should be addressed. Electronic addresses: a.abdelrahman@ecu.edu.au and hunskang@gist.ac.kr.
}

tions for excitonic quantum degenerate gases. ${ }^{9}$ In this article, we report on the observation of spatially distributed inhomogeneous depressions of the Zeeman splittings of the heavyhole excitons (HH-excitons) and light-hole excitons (LHexcitons) caused by an inhomogeneous magnetic field.

\section{MAGNETIC INDUCED SHIFT OF THE EXCITONS DISPERSION SURFACE IN THE $\kappa$-SPACE}

It is well known that the exciton exhibits a hydrogenlike nature because of its composite particle internal motion, in which case the electron and hole are mutually orbiting each other, plus the translational motion of their center of mass. Here, we assume that the ground state of the exciton's two-particle motion has a finite momentum, ${ }^{10,11}$ and the optically active states are those for free excitons with momentums close to zero. They recombine in a radiative zone, defined in the $k$-space by the intersection of the exciton dispersion surface $E_{\text {ext }}(P)$ with the excitation photon cone $E_{p h}=c P / r \sqrt{\varepsilon}$, where $\varepsilon$ is the dielectric constant. ${ }^{12-14} \mathrm{~A}$ schematic representation of the intersection of the exciton dispersion curve in the momentum-space is shown in Fig. 1. The exciton energy is defined as $E_{\text {ext }}(P)=-E_{B} e^{-\alpha} I_{o}(-\alpha)$, where $I_{o}(-\alpha)$ is the modified Bessel function and $\alpha=\left(P l_{B} / 2 \hbar\right)^{2}$, with $l_{B}$ being the magnetic length. ${ }^{15}$ The momentum operator $\mathrm{P}$ of the center of mass $(\mathrm{CM})$ in a magnetic field $\mathrm{B}$ is defined as $\mathrm{P}=M_{e x t} v_{c m}-\frac{e}{c} \mathrm{~B} \times \mathrm{x}_{e x t}$, where $\mathrm{x}_{e x t}=\mathrm{x}_{e}-\mathrm{x}_{h}$ is the relative coordinate of the excitonic electron-hole motion and $M_{e x t}$ is the effective mass of the exciton and $v_{c m}$ is the CM velocity.

In the system under investigation, the inhomogeneous magnetic cover is imprinted to lay at the quantum wells confining plane, where the magnetic field is generated by a semitransparent permanent magnetic material integrated with the multiple quantum wells system. The magnetic material is 


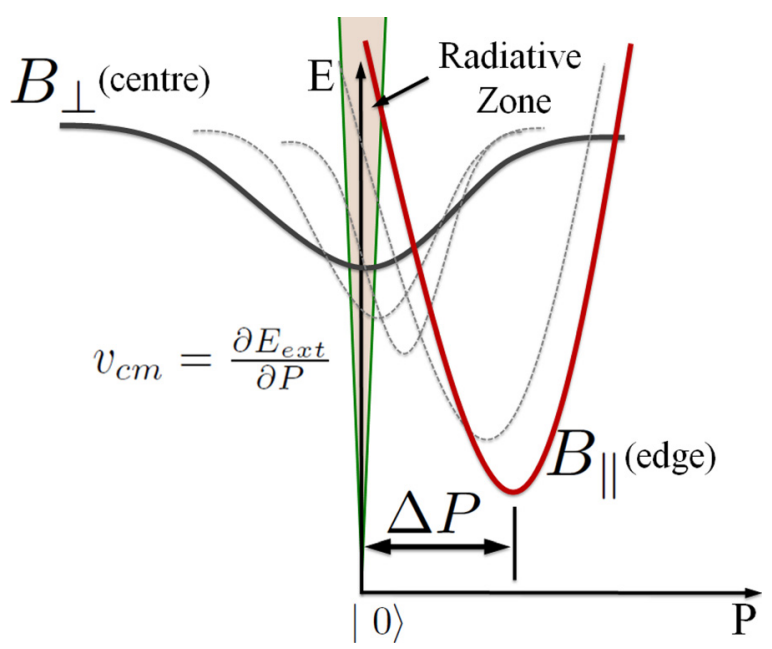

FIG. 1. (Color online) Schematic representation of the exciton dispersion curve shift in the $k$-space characterized by the center of mass velocity, $v_{c m}=\partial E_{\text {ext }} / \partial P$, where its intersect with the radiative zone (i.e., the photon cone $E_{p h}=c P(\sqrt{\varepsilon})$ also shifts along the energy direction due to the inhomogeneity of the magnetic field across the $\mathrm{x} / \mathrm{y}$ plane. The localized magnetic field varies from the region where the magnetic field is perpendicular $\left(B_{\perp}\right)$ to the $x / y$-plane of the sample at the center to a region where the field is pointing almost parallel $\left(B_{\|}\right)$to the $\mathrm{x} / \mathrm{y}$-plane at the edges.

uniaxially anisotropic and its magnetization vector is pointing in the $\mathrm{z}$-direction perpendicularly to the film $\mathrm{x} / \mathrm{y}$-plane. The inhomogeneity inherited in the field distribution is due to the edge effects, i.e., high field values occur at the edges. Consequently, a relatively low magnetic field value occurs at the center with a field direction that points almost perpendicular $\left(B_{\perp}\right)$ to the $x / y$-plane of the integrated sample; meanwhile, the field is nearly parallel to the $\mathrm{x} / \mathrm{y}$-plane $\left(B_{\|}\right)$at the edges, with high values compared to the center of the sample. ${ }^{16}$ Simulated magnetic fields of the permanently magnetized thin film are shown in Figs. 2(a)-2(c).

At the $B_{\perp}$ field region, the exciton states shift rigidly in $k$-space, in which case the recombination lifetime of the excitons is slightly modified, as will be shown in the results, by the localized inhomogeneous magnetic field without affecting their binding energies that defined as $E_{B}=\sqrt{\pi} / 2 e^{2} / \varepsilon l_{B} \sim$ $\sqrt{B_{\perp}}$, where $l_{B}=\sqrt{\hbar c / e B_{\perp}} \cdot{ }^{17}$ Thus, the migration of the dispersion surface in $k$-space is a function of the magnetic field and can be observed as changes in the transport properties of the excitons. For example, as we observed, at the zones where $B_{\perp}$ field is dominated, the dispersion curve departs away from the center of the photon cone in the $k$-space by $\Delta P=-\hbar k=e / c d B_{\perp}$, as illustrated in Fig. $1 .^{18}$

\section{EXPERIMENTAL SETUPS AND RESULTS}

The non-magnetic material gadolinium gallium garnet, $\mathrm{Gd}_{3} \mathrm{Ga}_{5} \mathrm{O}_{12}$, is deposited onto the surface of the GaAs/ $\mathrm{Al}_{0.35} \mathrm{Ga}_{0.65} \mathrm{As}$ multiple quantum wells system, in which case the thickness of nonmagnetic material controls the values of the magnetic field at the quantum wells location. Then, the permanent magnetic material $\mathrm{Bi}_{2} \mathrm{Dy}_{1} \mathrm{Fe}_{4} \mathrm{Ga}_{1} \mathrm{O}_{12}$ is deposited on the top of the non-magnetic material layer using rf-sputtering technique, annealed at $876.15 \mathrm{~K}$, and then the fabricated sample is permanently magnetized along the z-axis. The $\mathrm{GaAs} / \mathrm{Al}_{0.35} \mathrm{Ga}_{0.65} \mathrm{As}$ multiple quantum wells are grown on a GaAs substrate using molecular beam epitaxy where the sample contains twenty periods of $9 \mathrm{~nm}$ thickness of GaAs quantum wells and $20 \mathrm{~nm}$ thickness of AlGaAs barriers. Fig. 2(d) shows the integrated magnetic material with
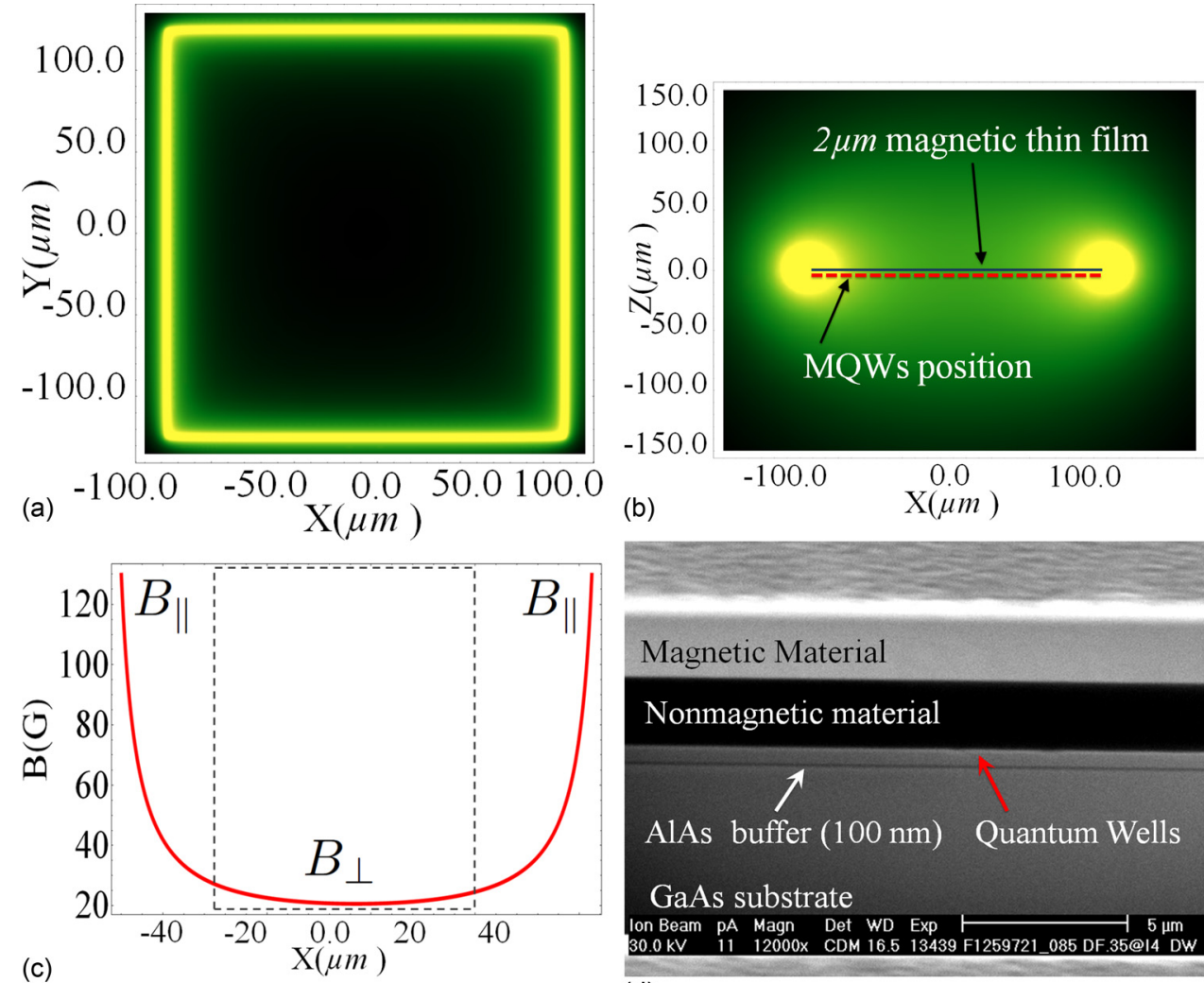

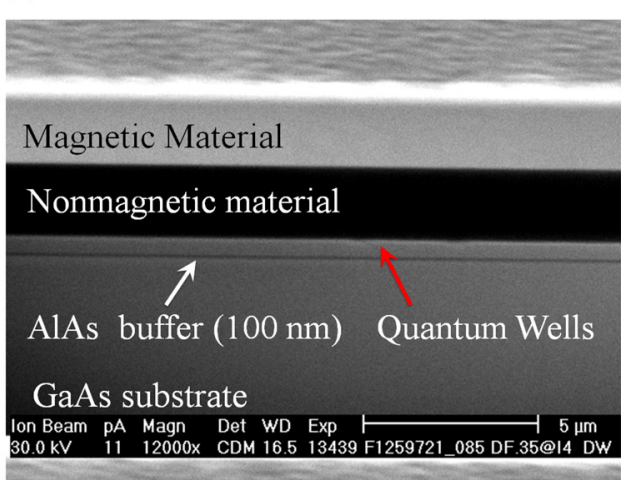

(d)
FIG. 2. (Color online) Density plot representations of the magnetic field magnitude across (a) the $x / y$-plane and (b) the $\mathrm{x} / \mathrm{z}$-plane of the integrated multiple quantum wells system. (c) Modeled magnetic field distribution along the $\mathrm{x}$-axis showing the edge effect. (d) A focused ion beam image illustrating the internal structure of the integrated magnetic-quantum well system. 
the multiple quantum well structure. The high absorption of the $\mathrm{Bi}_{2} \mathrm{Dy}_{1} \mathrm{Fe}_{4} \mathrm{Ga}_{1} \mathrm{O}_{12}$ magnetic material ${ }^{19}$ limits the excitation wavelengths to values above $600 \mathrm{~nm}$ when using the magnetic material top side to measure the photoluminescence (PL) signal. However, the former effect takes no place when using the bottom GaAs substrate side for excitation after removing a $200 \mu \mathrm{m}$ thickness from the substrate using mechanical polishing to avoid the absorption by the thick GaAs substrate when measuring the PL signal, thus, allowing that to use a $400 \mathrm{~nm}$ pulse for excitation.

The integrated magnetic multiple quantum wells sample is placed in an evacuated optical cryostat and cooled down in a helium path to $9 \mathrm{~K}$ to suppress the phonon interactions. In the first experimental setup, a Ti:sapphire laser pump, emitting a linearly polarized continuous wave $(\mathrm{CW})$ with photon energy of $\sim 1.724 \mathrm{eV}$, is used for excitation and delivered to the sample inside the optical cryostat via an automated pump scanning head fitted with a collimation system. The photoluminescence signal is collected and transferred via an optical fiber to a monochromator integrated with a Peltier cooled intensified charge-coupled device (ICCD) camera that is swept across the transition energy spectral region surrounding the optically active state of interest, i.e., $E \sim 1.547 \mathrm{eV}$. Then, the PL signal is fed electronically from a photomultiplier to be amplified and to eventually be monitored. The scanning head is used to scan lines within the $x / y$-plane with a step size of $2 \mu \mathrm{m}$, with the pump spot focused to $\sim 10 \mu \mathrm{m}$ size. In the second experimental setup, a time-correlated single-photon counting (TCSPC) scheme is used to measure the lifetimes of the $\mathrm{HH}$ and $\mathrm{LH}-$ excitons, which are excited using a right circularly polarized $\left(\sigma^{+}\right)$femtosecond laser with emission of $150 \mathrm{fs}$ pulse width and $76 \mathrm{MHz}$ repetition rate. The approximated delay time $\tau$ is presented in Table I, where these results are obtained by fitting the photoluminescence decay curves in Fig. 5. The values of $\tau_{1}$ indicate that the decay time increases when the excitons experience an induced shift in the dispersion due to the magnetic field while the recombination rate is decreased.

The data is collected by spatially resolving the PL signal across the $x / y$-plane of the sample, starting from the sample center toward the edges. Fig. 3(a) shows enhanced emissions

TABLE I. The delay time measured for HH-excitons and LH-excitons using linearly polarized light and circularly polarized light $\left(\sigma^{+}\right)$with the experimental conditions as specified in the text. The photoluminescence decay curves in Figure 5 are fitted using the PicoQuant FluoFit software with $n=2$ in the intensity approximation $I(t) \sim{ }_{i=1}^{n} e^{-\frac{t}{\tau_{i}}}$.

\begin{tabular}{llccc}
\hline \hline $\begin{array}{l}\text { Excitation } \\
\text { polarization }\end{array}$ & Position & $\begin{array}{c}\text { Exciton } \\
\text { type }\end{array}$ & $\begin{array}{c}\text { Delay time } \\
\tau_{1}(\mathrm{ps})\end{array}$ & $\begin{array}{c}\text { Delay time } \\
\tau_{2}(\mathrm{~ns})\end{array}$ \\
\hline \multicolumn{1}{c}{$\begin{array}{l}\text { Linear } \\
\text { polarization }\end{array}$} & Center & HH & $425 \pm 23$ & $3.024 \pm 0.074$ \\
& & LH & $419 \pm 159$ & $3.507 \pm 0.324$ \\
& Edge & HH & $468 \pm 10$ & $2.503 \pm 0.027$ \\
Right circular & Center & LH & $501 \pm 76$ & $3.302 \pm 0.112$ \\
polarization $\sigma^{+}$ & & LH & $448 \pm 11$ & $3.297 \pm 0.024$ \\
& Edge & HH & $560 \pm 36$ & $3.357 \pm 0.070$ \\
& & LH & $556 \pm 34$ & $3.476 \pm 0.012$ \\
& & & &
\end{tabular}

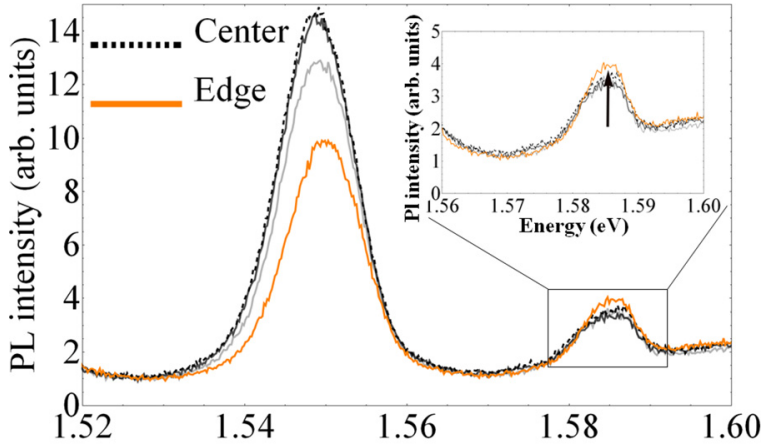

(a) Energy $(\mathrm{eV})$

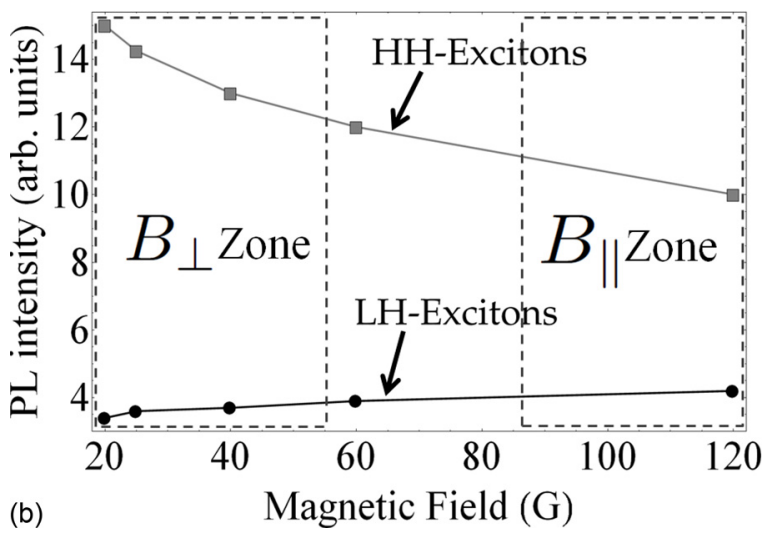

FIG. 3. (Color online) (a) Measured photoluminescence spectra across the x/y-plane, starting from the perpendicular magnetic field $B_{\perp}$ zone at the center toward the parallel $B_{\|}$field zone at the edges of the sample. A linearly polarized continuous wave (CW) excitation pump of energy $\sim 1.724 \mathrm{eV}$ is used, and the emission is probed at $\sim 1.55 \mathrm{eV}$. The inset shows the spatially resolved conversion of the LH-excitons. (b) Extracted PL maxima (related to peaks energy) from Fig. 3(a), which shows two different regions of two different optically active states of $\mathrm{HH}$ and $\mathrm{LH}$-excitons, where these results show that the energy transition from the initial HH-exciton states $\left(\left|\frac{ \pm 3}{2}, \frac{\mp 1}{2}\right\rangle\right)$ and initial LH-exciton states $\left(\left|\frac{ \pm 1}{2}, \frac{\mp 1}{2}\right\rangle\right)$ to the final states indicates that the initial states at $B_{\|}$zone (higher on energy scale) lies above the final states at $B_{\perp}$ zone (lower on energy scale).

around the HH-exciton energy point measured at the $B_{\perp}$ field region, where the intersection of the dispersion curve and the radiative zone is expected to shift in $k$-space with no change

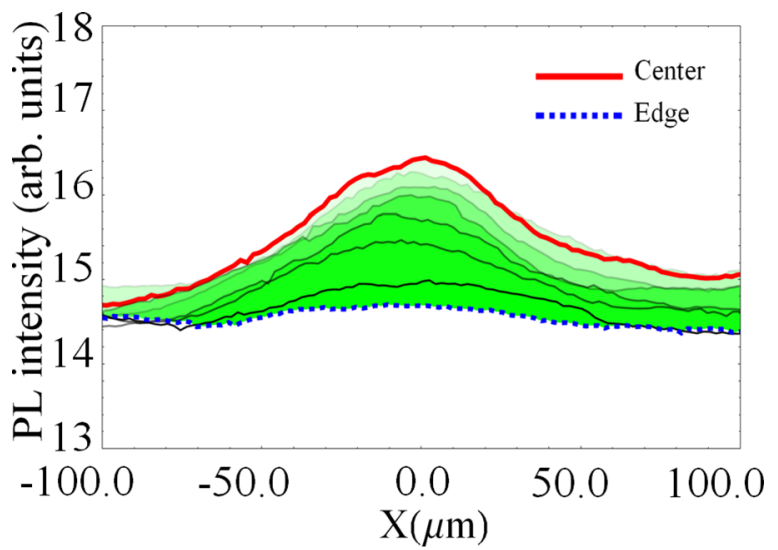

FIG. 4. (Color online) Spatially resolved PL spectra of energy $\sim 1.55 \mathrm{eV}$ corresponding to the optically active $\left\langle\frac{ \pm 3}{2}, \frac{\mp 1}{2}\right\rangle \mathrm{HH}$-exciton states shows higher density of HH-excitons at low field regions at the center compared to the edges. An automated focused-pump scanning head is used to scan the $x / y-$ plane with steps of $\sim 2.5 \mu \mathrm{m}$ along the $\mathrm{x}$-axis and $\sim 10 \mu \mathrm{m}$ along the $\mathrm{y}$-axis, using a linearly polarized $\mathrm{CW}$ excitation pump of energy $\sim 1.724 \mathrm{eV}$. 


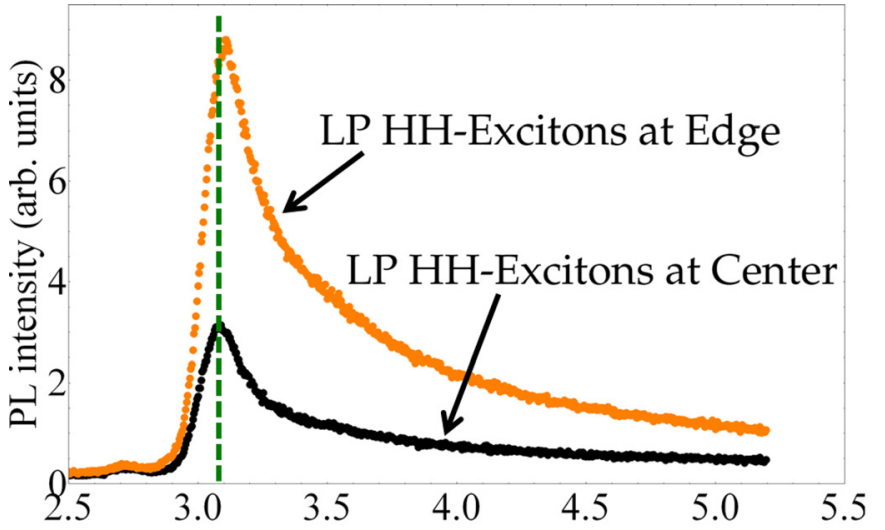

(a)

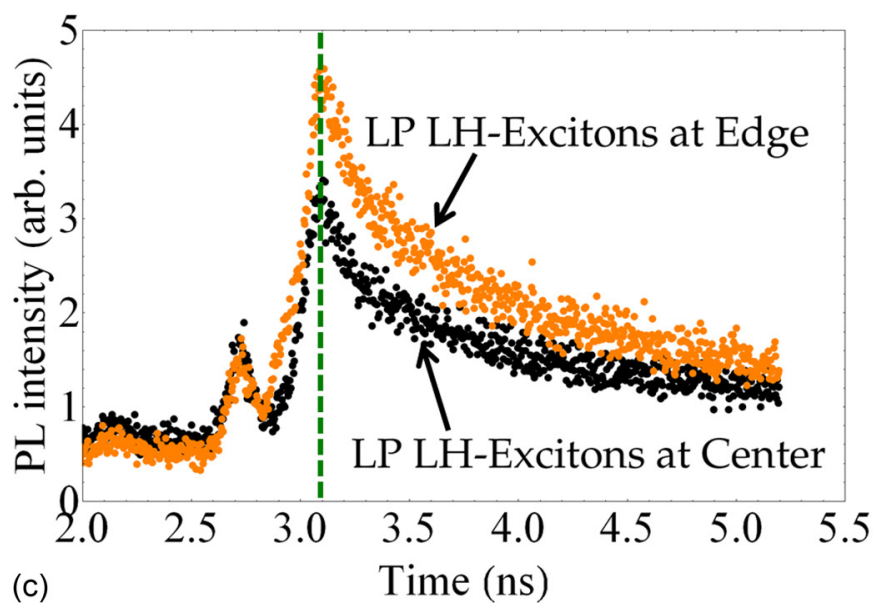

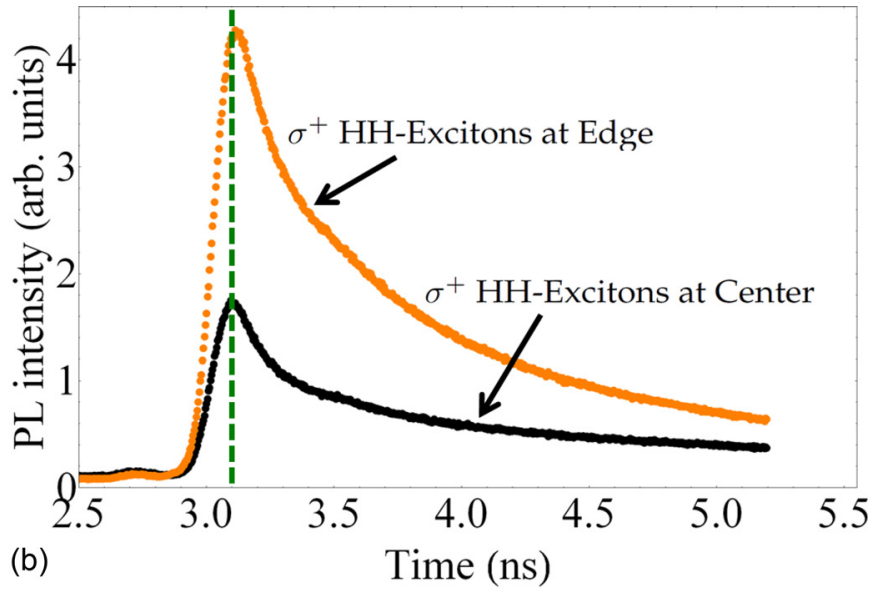

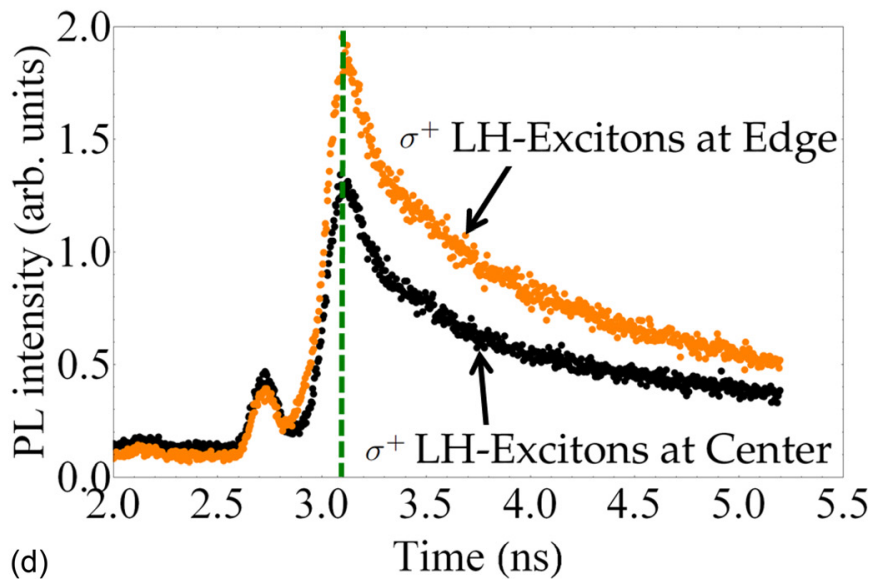

FIG. 5. (Color online) Time correlated single photon counting (TCSC) measurements of the PL decay show a spatial dependence of the exciton radiative decay rate with respect to the localized inhomogeneous magnetic field. A linearly polarized pump is used to excite (a) the HH-excitons and (c) the LH-excitons at different locations within the $B_{\perp}$ and $B_{\|}$regions, and a right circularly polarized $\sigma^{+}$pump is also used to create (b) the HH-excitons and (d) the LH-excitons. Dotted line indicates the excitation pulse.

along the momentum direction. However, due to the inhomogeneity of the localized magnetic field, a migration of the intersected zone across the $k$-space occurs toward a region where $B_{\perp}$ is dominant. This behavior can be identified as a shift in the energy peaks of HH-excitons occurring due to the depression of Zeeman splittings and a reduction in their PL intensities, as shown in Fig. 3(b). Meanwhile, as shown in the inset of Fig. 3(a), a detectable conversion between the HHexcitons and the LH-excitons occurs when scanning the PL signal across the $x y$ plane from the center toward the edges.

We believe that the spatial conversion is happening because the optically active states of the HH-excitons, $\left|\frac{ \pm 3}{2}, \frac{\mp 1}{2}\right\rangle$, and those of the LH-excitons, $\left|\frac{ \pm 1}{2}, \frac{\mp 1}{2}\right\rangle$, suffer slightly inverted modifications in their magnetic lengths (thus, depression in Zeeman splittings) depending on the value and the direction of the localized magnetic field, which itself gradually changes across the $x / y$-plane. The effect of the localized field on the LH-exciton is weak, as also reported by J. J. Davies et $a .^{2}$ using strong magnetic bias fields. Accumulation of the $\mathrm{HH}$-excitons at the center is shown in Fig. 4, in which case the scanning probe is adjusted to only record the emitted photons with energies surrounding the transition of the HH-exciton states, i.e., $E \sim 1.547 \mathrm{eV}$. As shown in Fig. 5, the PL decay processes slightly differ when measured at the two different radiative regions: the $B_{\perp}$ and
$B_{\|}$-dominated. The results shown in these figures are generated using right circularly polarized $\sigma^{+}$and linearly polarized excitation photons.

\section{CONCLUSION}

We show that an inhomogeneous spatial distribution of the excitonic density can be generated, and possibly configured, using a localized inhomogeneous weak magnetic field. Using the available fabrication technologies, one can integrate semi-transparent permanent magnetic materials with multiple quantum well systems and introduce an accessible degree of freedom to tune the characteristic (magnetic) properties of excitons. Spatially resolved photoluminescence measurements indicate an inverted momentum-space migration of $\mathrm{HH}$-excitons and LH-excitons in response to the profile inhomogeneity of the magnetic field. The experiments were conducted using relatively small quantum well widths (less than the bulk exciton Bohr radius of excitons in $\mathrm{GaAs} \sim 13.5 \mathrm{~nm})^{18}$ with wide barriers, limiting our observations to direct excitons only. However, it is rather interesting to carry out these studies for indirect excitons in multiple quantum wells with sufficiently wide widths and narrow barriers relative to the exciton Bohr radius and compared to their magnetic length, in which case we can expect the adiabatic approximation to be applicable and, hence, simplify some theoretical models. ${ }^{19,20}$ More 
importantly, specific magnetic field patterns can be projected through the surface of the magnetic material into the plane of the quantum wells, such as magnetic micro-trapping patterns in scenarios similar to that of magnetic trapping of cold atoms at very low temperatures. ${ }^{15,21,22}$

We thank Seong-Min Ma and Byoung S. Ham at Inha University, Republic of Korea for providing the GaAs/ $\mathrm{Al}_{0.35} \mathrm{Ga}_{0.65} \mathrm{As}$ multiple quantum wells sample.

${ }^{1}$ J. J. Davies, L. C. Smith, D. Wolverson, V. P. Kochereshko, J. Cibert, H. Mariette, H. Boukari, M. Wiater, G. Karczewski, T. Wojtowicz, A. Gust, C. Kruse, and D. Hommel, Phys. Status Solidi B 247, 1521 (2010).

${ }^{2}$ J. J. Davies, D. Wolverson, V. P. Kochereshko, A. V. Platonov, R. T. Cox, J. Cibert, H. Mariette, C. Bodin, C. Gourgon, E. V. Ubyivovk, Yu. P. Efimov, and S. A. Eliseev, Phys. Rev. Lett. 97, 187403 (2006).

${ }^{3}$ L. C. Smith, J. J. Davies, D. Wolverson, S. Crampin, R. T. Cox, J. Cibert, H. Mariette, V. P. Kochereshko, M. Wiater, G. Karczewski, and T. Wojtowicz, Phys. Rev. B 78, 085204 (2008).

${ }^{4}$ L. Butov, C. Lai, D. S. Chemla, Y. E. Lozovik, K. L. Campman, and A. C. Gossard, Phys. Rev. Lett. 87, 216804 (2001).

${ }^{5}$ J. A. K. Freire, F. M. Peeters, A. Matulis, V. N. Freire, and G. A. Farias, Phys. Rev. B 62, 7316 (2000).

${ }^{6}$ M. A. McCord and D. D. Awschalom, Appl. Phys. Lett. 57, 2153 (1990).
${ }^{7}$ J. A. K. Freire, A. Matulis, F. M. Peeters, V. N. Freire, and G. A. Farias, Phys. Rev. B 61, 2895 (2000).

${ }^{8}$ J. Levy, V. Nikitin, J. M. Kikkawa, A. Cohen, N. Samarth, R. Garcia, and D. D. Awschalom, Phys. Rev. Lett. 76, 1948 (1996).

${ }^{9}$ Y. Kuramoto and C. Horie, Solid State Commun. 25, 713 (1978).

${ }^{10}$ D. Paquet, T. M. Rice, and K. Ueda, Phys. Rev. B 32, 5208 (1985).

${ }^{11}$ A. Imamoglu, Phys. Rev. B 54, 14285 (1996).

${ }^{12}$ J. Feldmann, G. Peter, E. Göbel, P. Dawson, K. Moore, C. Foxon, and R. J. Elliott, Phys. Rev. Lett. 59, 2337 (1987).

${ }^{13}$ A. Parlangeli, P. C. M. Christianen, J. C. Maan, I. V. Tokatly, C. B. Soerensen, and P. E. Lindelof, Phys. Rev. B 62, 15323 (2000).

${ }^{14}$ S. Charbonneau, M. L. W. Thewalt, E. S. Koteles, and B. Elman, Phys. Rev. B 38, 6287 (1988).

${ }^{15}$ A. Abdelrahman, M. Vasiliev, K. Alameh, and P. Hannaford, Phys. Rev. A 82, 012320 (2010).

${ }^{16}$ A. A. Gorbatsevich and I. V. Tokatly, Semicond. Sci. Technol. 13, 288 (1998).

${ }^{17}$ P. C. Wo, P. R. Munroe, M. Vasiliev, Z. H. Xie, K. Alameh, and V. Kotov, Opt. Mater. 32, 315 (2009).

${ }^{18}$ R. Binder and M. Lindberg, Phys. Rev. Lett. 81, 1477 (1998).

${ }^{19}$ M. Altarelli and N. O. Lipari, Phys. Rev. B 7, 3798 (1973).

${ }^{20}$ K. Cho, S. Suga, W. Deybrodt, and F. Willman, Phys. Rev. B 11, 1512 (1975).

${ }^{21}$ R. M. A. S. M. Singh and P. Hannaford, Phys. Rev. A 79, 053407 (2009).

${ }^{22}$ S. Whitlock, R. Gerritsma, T. Fernholz, and R. Spreeuw, New J. Phys. 11, 023021 (2009). 